\section{Prehospital Care in Rural Settings}

Attila J. Hertelendy, BHsc., CCEMT-P, NREMT-P

University of Mississippi Medical Center, USA

Oxford, Mississippi USA

The delivery of prehospital care in a rural setting requires a conceptual framework quite different from that required in urban and suburban environments, given that available resources are limited in the rural setting. The intermittent and episodic nature of seriously ill and injured patients who present to rural emergency medical services (EMS) makes it difficult to plan, staff, and equip in order to provide emergency medical care at the same level seen at highervolume urban or suburban institutions. The objective of this presentation is to describe the distinctive nature and widely unrecognized features of prehospital care in rural and remote areas, with a focus on clinical, workforce, and economic issues, through a Canadian perspective that adds the element of extreme temperatures. The author presents recommendations for a paradigm shift in thinking, and a call to action on behalf of all prehospital care professionals that are based on a realistic assessment of the current status of emergency medicine, and that are needed to develop and sustain high-quality emergency medical care in rural environments.

Keywords: emergency medical services (EMS); emergency medicine; medical care; prehospital; quality; remote areas; rural; suburban; urban Prehosp Disast Med 2002;17(s2):s77.

Major Incidents in Region Västra Götaland, Sweden, during 25 years

Annika Hedelin, RN; Iréne Johansson, Education

Secretary; Birger Kollberg, Official Reports Secretary

Centre for Prehospital and Disaster Medicine, Gothenburg,

Sweden

Region Västra Götaland was created in 1999 by merging three former county councils and Göteborg's decisionmaking functions concerned with healthcare. The region's main responsibility is to provide its 1.5 million inhabitants with adequate healthcare. Västra Götaland, together with Stockholm and Malmö, are the areas with the highest population densities in Sweden. The infrastructure is complex with high industrial activity and intense communication networks.

Over the last 20 years the region has had several major incidents, for example:

\begin{tabular}{|c|c|c|c|}
\hline Year & Incident & Dead & Injured \\
\hline 1977 & Landslide in Tuve & 9 & 62 \\
\hline 1980 & Bridge collapse, Tjörn & 8 & \\
\hline 1987 & Train accident, Lerum & 9 & 130 \\
\hline \multirow[t]{2}{*}{1990} & Fire on a passenger ship, & & \\
\hline & Scandinavian Star & 158 & 30 \\
\hline 1992 & $\begin{array}{l}\text { Tram accident in } \\
\text { Gothenburg }\end{array}$ & 13 & 37 \\
\hline 1998 & $\begin{array}{l}\text { Fire at a discotheque, } \\
\text { Gothenburg }\end{array}$ & 63 & 213 \\
\hline 1999 & $\begin{array}{l}\text { Fire on a passenger ship, } \\
\text { Princess Ragnhild }\end{array}$ & 1 & 10 \\
\hline \multirow[t]{2}{*}{2002} & $\begin{array}{l}\text { EU meeting, Gothenburg } \\
\text { Gas leak, Skara }\end{array}$ & 143 & \\
\hline & Sommarland & 39 to & \\
\hline
\end{tabular}

Questions:
How can we prevent such events?

What conclusions can we make?

What have we learned?

Keywords: communications; incidents, major; infrastructure; injured; lessons learned; networks; outcomes; prevention; Sweden

Prehosp Disast Med 2002;17(s2):s77-78.

\section{See What's Going On at the Scene: Remote Controllable, Mobile Video System Using a Cellular Phone}

Hideo Tohira; Jun'ichiro Yokota

Senshu Critical Care Medical Center, Osaka, Japan

Introduction: At the time of a disaster, the information from the scene is very limited. If we can see what is going on at the scene or in the ambulance, it may be useful for preparation of the emergency department.

Purpose: To create a remote controllable, mobile video system.

Method: We built up the mobile video system in an ambulance. The equipment on the ambulance includes: (1) a control unit; (2) MediaPoint mobile (NEC Corporation, Tokyo); (3) a remote controllable camera, EVI-D30, (SONY Corporation, Tokyo); and (4) a cellular phone. The camera is controlled by a computer in a remote hospital, and the moving image is transferred to the computer via a cellular phone. The image produced was a slow-moving image at ten frames per second.

Results: Using this system, the direction and magnification of the camera could be controlled without any trouble to the crew on the ambulance, and images of the inside of ambulance could be seen. It was possible to collect a variety of visual information in the ambulance. The information included: (1) The time the patient was loaded into the ambulance; (2) the time and the way a patient was intubated; (3) the medication used; and so on. From this information, we could prepare the ventilator and drugs prior to arrival of the patient.

Conclusion: This system is very helpful in preparing the emergency department at the time of emergency and possibly at the time of disaster.

Keywords: ambulance; cellular phone; emergency department; images; remote control; times; video

Prebosp Disast Med 2002;17(s2):s77.

Influence of Levels of SOD, MDA, and NO in Plasma and Tissues by Closed-Chest Hypothermia Cardiopulmonary Bypass (CPB) during Reperfusion Injury

Pei-zhi Huang;, ${ }^{1}$ Zhou Guang-Hua, ${ }^{2}$ Cheng An-Qing;, Wu ke-Jian, ${ }^{3}$ Wan Zhe ${ }^{2}$ Liu Zhu-Yi, ${ }^{3}$ Gu Jian-Yong, ${ }^{1}$ Gu Guo-Rong, ${ }^{1}$ Li Ying-Chuan ${ }^{1}$

1. Emergency Department

2. Cardiac Surgery Department

3. Department of Anesthesiology

Zhongshan Hospital, Fudan University, Shanghai, China

Objective: To observe levels of superoxide dismutase (SOD), malondialdehyde (MDA), and nitrogen monoxide (NO) resulting from mild or deep hypothermia after cardiac arrest of 15 minutes in dogs.

Method: With our established dog cardiopulmonary 